Planetary Systems in the Universe - Observation, Formation and Evolution

Proceedings IAU Symposium No. 202, (C)2004 IAU

Alan Penny, Pawel Artymowicz, Anne-Marie Lagrange, 8 Sara Russell, eds.

\title{
Timescales of Disk Evolution and Planet Formation
}

\author{
Ray Jayawardhana \\ Department of Astronomy, University of California, Berkeley, \\ CA 94720, U.S.A.
}

\begin{abstract}
It has been suggested that circumstellar disks evolve from dense, actively accreting structures to low-mass, replenished remnants. During this transition, grains may assemble into planetesimals, or the disk may be cleared by newborn planets. Recently identified nearby groups of young stars provide valuable laboratories for probing disk evolution. I discuss the properties of dust disks in the TW Hydrae Association and the MBM 12 cloud, and compare the results to other studies of disk evolution and planet formation timescales.
\end{abstract}

\section{Introduction}

Planetary systems form out of circumstellar disks that are the remnants of star formation (Shu, Adams, \& Lizano 1987). The young T Tauri stars with ages of $\sim 1 \mathrm{Myr}$, which frequently have optically-thick, actively-accreting disks (Strom, Edwards \& Skrutskie 1993), are thought to represent a stage prior to the main epoch of planet formation. In contrast, the dust disks of the so-called Vega-like objects, main sequence stars whose ages could be as large as $1 \mathrm{Gyr}$, appear to be more evolved; most of the dust has presumably coagulated into planets or planetesimals, and the remaining dust in the optically-thin disk is continually replenished by collisions between larger bodies (Lagrange, Backman \& Artymowicz 2000). However, the age at which this transition occurs is not well constrained. One reason is a paucity of intermediate-age $(t \sim 10 \mathrm{Myr})$ nearby stars in current samples. Unfortunately, the age estimates of early-type isolated main sequence stars are highly uncertain.

The two competing theories for making gas giant planets in disks predict very different timescales. Core accretion mechanism, in which a solid core builds up from icy and rocky planetesimals and later acquires an envelope of nebular gas, is expected to take $\sim 10 \mathrm{Myr}$ (e.g., Pollack et al. 1996). On the other hand, the disk instability mechanism, where a gravitationally unstable disk fragments directly into self-gravitating protoplanetary clumps, is a much faster process, forming giant planets in $\sim 0.1 \mathrm{Myr}$ (e.g., Boss 2000). Therefore, better observational constraints on disk evolution timescales may allow us to distinguish between the two scenarios.

\section{Insights from Nearby Stellar Groups}

The ROSAT all-sky X-ray survey, combined with follow-up spectroscopy and proper motion data, has led to the recent identification of several groups of young stars within $100 \mathrm{pc}$ of the sun. The proximity and age differences of these 
groups make them suitable laboratories for detailed studies of disk evolution and planet formation.

The TW Hydrae Association (TWA), at a distance of $\sim 55 \mathrm{pc}$ and not associated with any obvious parent cloud, is of particular interest because of its $\sim 10-\mathrm{Myr}$ age, as estimated using a variety of techniques. Our mid-infrared observations, carried out using the OSCIR instrument on Keck and the Cerro Tololo 4-meter telescope over the past two years, show that many of the TWA stars have little or no disk emission at $10 \mu \mathrm{m}$ (Jayawardhana et al. 1999b). Even among the five stellar systems with $10 \mu \mathrm{m}$ excesses, most show some evidence of inner disk evolution. The disk around the A0V star HR 4796A has an $r \approx 50 \mathrm{AU}$ central hole in mid-infrared images (Jayawardhana et al. 1998; Koerner et al. 1998). The SEDs of HD 98800 and Hen 3-600A also suggest possible inner disk holes (Jayawardhana et al. 1999a). The modest excess we detected from CD $-33^{\circ} 7795$ could well be due to a faint companion. Only TW Hya itself appears to harbor an optically thick, actively accreting disk of the kind observed in $\sim 1$ Myr-old classical T Tauri stars; it is the only one with a large $\mathrm{H} \alpha$ equivalent width $(-220 \AA)$.

If the TWA stars are indeed 10 million years old, our results suggest that their inner disks have already depleted either through coagulation of dust or accretion on to the central star. The fact that only one (TW Hya) out of a sample of 16 TWA members shows classical T Tauri characteristics-compared to $\sim 50 \%-90 \%$ of $\sim 1$-Myr-old stars in star-forming regions- argues for relatively rapid evolution of inner disks in pre-main-sequence (PMS) stars. Observations at far-infrared and sub-millimeter wavelengths may reveal whether most TWA stars still retain their outer disks.

We have also obtained mid-infrared photometry and high-resolution optical spectra for eight confirmed members of the MBM 12 high-latitude cloud at $\sim 65$ pc. These stars are estimated to be 1-3 Myr in age. Interestingly, in contrast to the TWA sample, the majority of MBM 12 stars do show signatures of optically thick, actively accreting disks (Jayawardhana et al. 2000).

\section{Comparison to Other Studies}

Our results for TWA and MBM 12 are consistent with those of Skrutskie et al. (1990) who studied infrared excess in a sample of "solar-type" PMS stars in Taurus-Auriga. Of stars estimated to be younger than $3 \mathrm{Myr}$, Skrutskie et al. found that roughly half have optically thick disks extending close to the stellar surface whereas fewer than $10 \%$ of somewhat older stars showed evidence for similar disks. Therefore, the authors suggested that few disks survive beyond 10 Myr.

More recently, Brandner et al. (2000) have compared disk properties between Chamaeleon and Sco-Cen star-forming regions to derive disk evolution timescales. They find that the 5-15 Myr-old stars in Sco-Cen have a $15 \mu \mathrm{m} / 7 \mu \mathrm{m}$ spectral index intermediate between that of younger (1-5 Myr-old) Chamaeleon objects and that of pure stellar photospheres. Brandner et al. suggest that the difference is due to a deficiency of small dust grains relative to large dust grains in the Sco-Cen disks. If it is an evolutionary effect, dust depletion of disks must start at an age between 5 and $15 \mathrm{Myr}$, again in agreement with our findings for 
the TWA and MBM 12 groups. Meteoritic evidence also suggests that planetary material was made over a period of $\sim 10 \mathrm{Myr}$ in the solar nebula (Podosek \& Cassen 1994; Wadhwa \& Russell 2000).

On the other hand, in high-mass star-forming regions such as Orion, disks may dissipate much faster (in $<1 \mathrm{Myr}$ ) due to irradiation from neighboring luminous stars (e.g., Bally et al. 1998). It is not clear whether planets can form in such rapidly photoevaporating disks.

\section{Future Prospects}

It is important to study a sufficiently large sample of stars with a range of ages and environments to place strong constraints on disk evolution and planet formation timescales. The identification of additional groups of young stars in the solar neighborhood will help, as will the unprecedented sensitivity of SIRTF and SOFIA to detect even optically thin disk emission from clusters at much greater distances than hitherto possible. There may not be a universal timescale for disk evolution, especially when the effects of binary companions and external irradiation are taken into account. More studies are also needed to better understand the dissipation of the gaseous component of disks and to determine whether it persists long enough to allow for slow accumulation of gas onto giant protoplanets, as predicted by the core accretion scenario.

\section{References}

Bally, J., Testi, L., Sargent, A., \& Carlstrom, J. 1998, AJ, 116, 854

Boss, A.P. 2000, ApJ, 536, L101

Brandner, W., et al. 2000, AJ, 120, 950

Jayawardhana, R., et al. 1998, ApJ, 503, L79

Jayawardhana, R., et al. 1999a, ApJ, 520, L41

Jayawardhana, R., et al. 1999b, ApJ, 521, L129

Jayawardhana, R., et al. 2000, in preparation

Koerner, D., et al. 1998, ApJ, 503, L83

Lagrange, A.-M., Backman, D.E., \& Artymowicz, P. 2000, in Protostars and Planets IV, ed. V. Mannings, A.P. Boss, \& S.S. Russell (Tucson: University of Arizona Press), 639

Podosek, F.A., \& Cassen, P. 1994 , Meteoritics, 29, 6

Pollack, J. B., et al. 1996, Icarus, 124, 62

Shu, F.H., Adams, F.C., \& Lizano, S. 1987, ARA\&A, 25, 23

Skrutskie, M.F., et al. 1990, AJ, 99, 1187

Strom, S.E., Edwards, S., \& Skrutskie, M.F. 1993, in Protostars and Planets III, ed. E.H. Levy \& J.I. Lunine (Tucson: Univ. Arizona Press), 837

Wadhwa, M., \& Russell, S.S. 2000 in Protostars and Planets IV, ed. V. Mannings, A.P. Boss, \& S.S. Russell (Tucson: University of Arizona Press), 995 